\title{
Automatic calculation of the dynamic characteristics of the manipulator on the basis of euler - lagrange equation
}

\author{
Alexander Goritov \\ the Department of ACS \\ Tomsk state University of control system and \\ Radioelectronics \\ Tomsk \\ ang@asu.tusur.ru
}

\begin{abstract}
Robotics is very popular in today's world. The robots are widely used in various fields of human life. They are used in medicine for development of space in the field of safety at work and at home. However, the design and simulation of the robot is a long and tedious process. Therefore, special programs developed for more efficient operation. They make it possible to create a model of the robot. For example, automated system for modeling RAUMS controlled mechanical systems. RAUMS simulation system modification is discussed in the report. A modification of the system makes it possible to perform calculations of the dynamic characteristics of the examined objects. The modification includes modification of the model components and the incorporation into the system in terms of the dynamic characteristics of the manipulator. The algorithm is based on the Euler-Lagrange method, and makes it possible to perform direct and inverse problem of dynamics. The result of the calculations is presented in the form of a table and a plot of dynamic characteristics in time. As a result of the development of the module have been expanded to simulation analysis in terms of the dynamic characteristics of the robot.
\end{abstract}

Keywords - Robot - manipulator, direct problem of dynamics, inverse dynamic problem, Lagrange - Euler method, RAUMS system.

\section{INTRODUCTION}

Robotics is very popular in today 's world. The robots are widely used in various fields of human life. They are used in medicine for development of space in the field of safety at work and at home. However, the design and simulation of the robot is fairly long and tedious process. Therefore, special programs developed for more efficient operation. They make it possible to create a model of the robot. For example, automated system for modeling RAUMS controlled mechanical systems. RAUMS enables simulation of the robot system and the external environment. It solves many tasks: control no collisions, movement at predetermined spatial constraints, check to execute assimilability conditions and searching for optimal layout decisions $[1,2]$. Dynamic characteristics of the examined object is important in designing.

\author{
Maria Molokova \\ the Department of ACS \\ Tomsk state University of control system and \\ Radioelectronics \\ Tomsk \\ masha_molokova@mail.ru
}

1. FORMULATION OF THE PROBLEM

The purpose of operation consists in designing the unit for calculating the dynamic characteristics of the manipulator and its incorporation into the system RAUMS, would expand the set of problems solved RAUMS simulation system.

In order to accomplish the above object, it is necessary to: analyze the task decision methods of direct and inverse dynamics, explore RAUMS, develop a system structure in terms of the dynamic characteristics of the system and integrate the developed block RAUMS

\section{THEORY}

\section{A. Algorithm for solving the direct and inverse problem of dynamics}

The dynamic-mechanical section, which are the cause of mechanical motion. The driver operates the concepts of mass force pulse energy. There are two main tasks: direct and inverse dynamics. Direct problem dynamics: according to a predetermined motion pattern to determine force acting on the body. The inverse problem: dynamics of predetermined forces to determine the nature of the motion of the body. There are several methods that calculate the Euler-Lagrange equation of dynamics, Gaussian [3], Newton-Euler [4]. Each of the methods has its own features. The Euler-Lagrange method allows convenient for analyzing the form of equations of motion. The Newton-Euler method makes it possible to obtain an extremely efficient system of equations computationally. The Gaussian method is an iterative method for solving linear equations is obtained. After the comparative analysis methods, the Euler-Lagrange method was selected. Since not only is important to us decision accuracy, as well as the computation time and the form of the equation .

The Euler-Lagrange equation for finding the dynamic characteristics is represented by the use (1). 


$$
\frac{d}{d t}\left[\frac{\partial L}{\partial \phi_{i}}\right]-\frac{\partial L}{\partial q_{i}}=\tau_{i}
$$

In use (1), L Lagrange function $\mathrm{L}=\mathrm{K}-\mathrm{P}, \mathrm{K}$ is the total kinetic energy of manipulator, $P$ is the total potential energy of the manipulator, $q_{i}$ generic-manipulator variables, $\dot{q}_{i}$ - the first derivative with respect to time of the generalized variables, $\tau_{i}$ generalized forces or moments $i$ link for which calculations are made.

Use (2) describes the dynamics of the robot movement in vector form:

$$
\tau_{i}=\sum_{k=1}^{n} D_{i, k} \&(t)_{k}+\sum_{k=1}^{n} \sum_{m=1}^{n} h_{i, k, m} \&(t)_{k} \&(t)_{m}-c_{i},
$$

where $\tau_{i}$ is the generalized forces $D_{i, k}$ is given by the use (3), establishes the forces and moments acting at joints with accelerations variables is attached $h_{i, k, m}$ is determined by use (4) and (5), establishes the forces and moments acting at joints with the rates of change of variables is attached $c_{i}$ is determined by the use (6), takes account of the force of gravity acting on each of the links of the manipulator, $)_{k}$ generic-acceleration $\mathscr{L}(t)_{m}$ is a generalized rate $n$-the number of links of the robotmanipulator.

The coefficients $D_{i k}$ are determined by the equationin

$$
D_{i k}=\sum_{j=\max (i, k)}^{n} \operatorname{Tr}\left(U_{j k} J_{j} U_{j i}^{T}\right) i, k=1,2, \ldots n .
$$

The coefficients $h_{i k j}$ are given by

$$
\begin{gathered}
h_{i k j}=\sum_{j=\max (i, k, m)}^{n} \operatorname{Tr}\left(U_{j k m} J_{j} U_{j i}^{T}\right) i, k, m=1,2, \ldots, n . \\
h_{i}=\sum_{k=1}^{n} \sum_{m=1}^{n} h_{i k m} \boldsymbol{\Phi}_{k}(t) \boldsymbol{\bigotimes}_{m}(t) i=1,2, \ldots n .
\end{gathered}
$$

where the matrix $U_{j i}^{j}$ describing the link $i$-th position change caused by the movement of the manipulator joint $j$-ohms, $J_{j}$ matrix inertia moments $i$-th link, $\operatorname{Tr}$ the trace of the matrix obtained.

The coefficients $c_{i}$ determined by the equation

$$
c_{i}=\sum_{j=i}^{n}\left(-m_{j} g U_{j i}^{j} \bar{r}_{j}\right) i=1 \ldots n
$$

where $m_{j}$ mass $j$-link $U_{j i}^{j}$ matrix describing $i$ the link position change caused by the movement of the manipulator $j$ joint, $\bar{r}_{j}$ vector mass center $i$ link in the home reference system, $g$ gravity acting on each of the links of the manipulator [3].

Use (2) assumes the following form for solving the inverse problem of dynamics :

$$
t_{i}=\frac{\left(\tau_{i}-\sum_{k=1}^{n} \sum_{m=1}^{n} h_{i k m} \&_{k} \&_{m}-c_{i}\right)}{\sum_{k=1}^{n} D_{i, k}}, i=\overline{1, n}
$$

The generalized variable and its derivative are calculated by use (8) and (9) when solving the inverse problem of dynamics.

$$
\begin{aligned}
& \phi(t+\Delta t)_{i}=\phi(t)_{i}+\phi(t)_{i} \cdot \Delta t, i=\overline{1, n} \\
& q(t+\Delta t)_{i}=q(t)_{i}+\phi(t)_{i} \cdot \Delta t, i=\overline{1, n}
\end{aligned}
$$

where $\Delta t$ is the time change.

The algorithm for solving the common forward and reverse dynamics problem is illustrated Fig. 1.

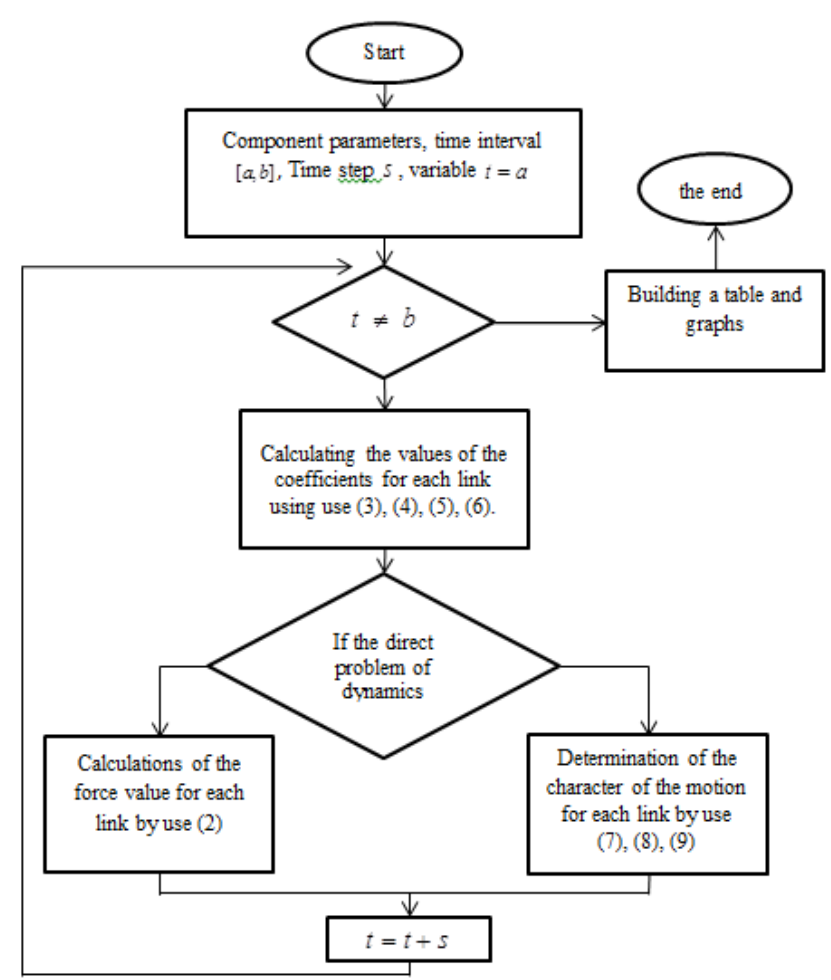

Fig. 1 Total algorithm for solving the problems

The following algorithm was implemented on the basis of the dynamics of the module. 


\section{EXPERIMENTAL RESULTS}

Referring to the operation of finding the dynamic characteristics of the module, by way of example. Given a manipulator, which consists of 4 units. The manipulator is shown in Fig. 2. Its kinematic diagram is given in Fig. 3.

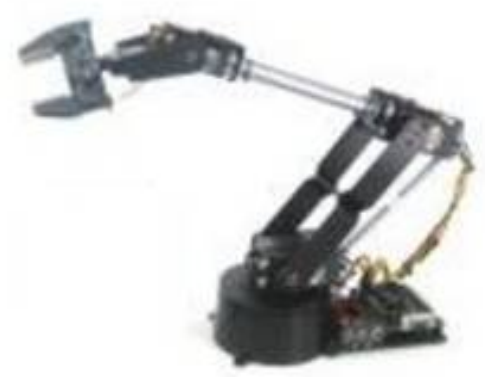

Fig. 2 Model of the manipulator

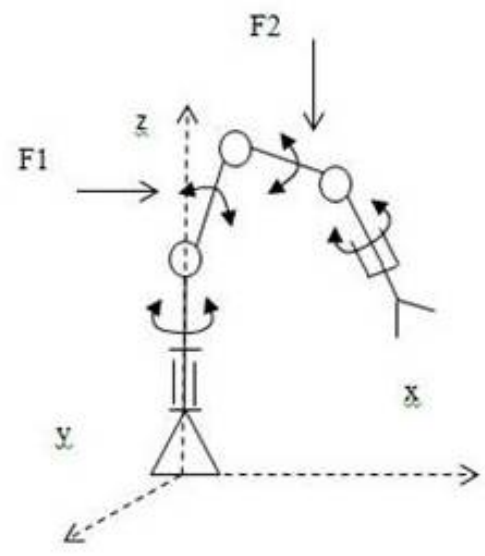

Fig. 3 The kinematics of the manipulator

The first link is rotational about the z-axis, its mass is 161.1 grams. The second and third links specify rotations about the $\mathrm{x}$ axis, the mass of each of them is equal to the mass of 85.5 grams. The fourth link is the grasp, it rotates around the y axis, its mass is 60 grams.

Fig. 4 and 5 show the manipulator model at different times. In Fig. 4 shows the initial position of the manipulator at the time. In Fig. 5 shows the final position of the manipulator at the time.

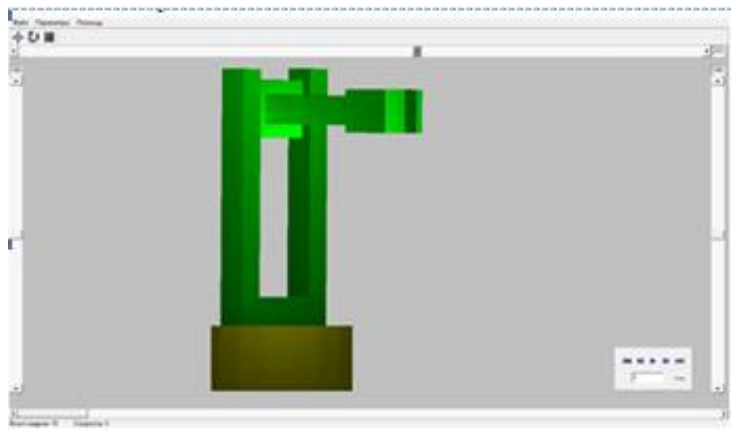

Fig. 4 Position of the manipulator at the initial moment of time

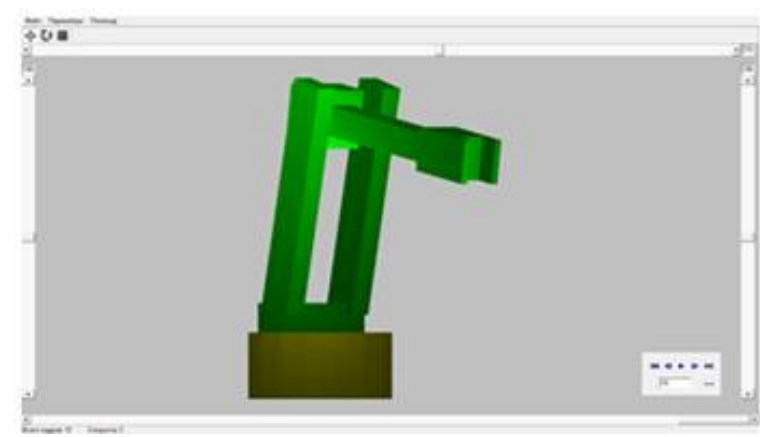

Fig. 5 Position of the manipulator at the end point of time

We use the constructed model to solve the direct and inverse problem of dynamics.

Direct problem. It is necessary to determine the forces that will be required to move the manipulator grasp from the initial position of Fig. 4 into the final fig. 5. At intervals of 0 to 1 second, in 0.1 second increments. The results of solving the direct problem of dynamics are presented in the form of the table in Fig. 6 and the graph of Fig. 7.

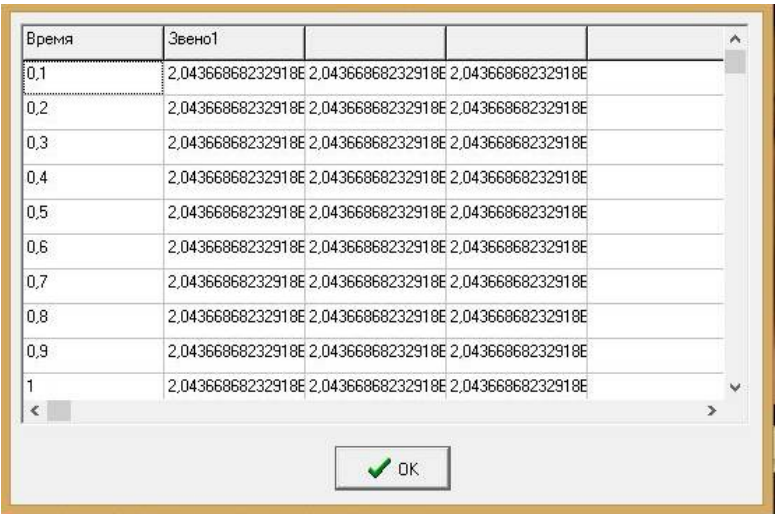

Fig. 6 Force acting on the gripper arm 


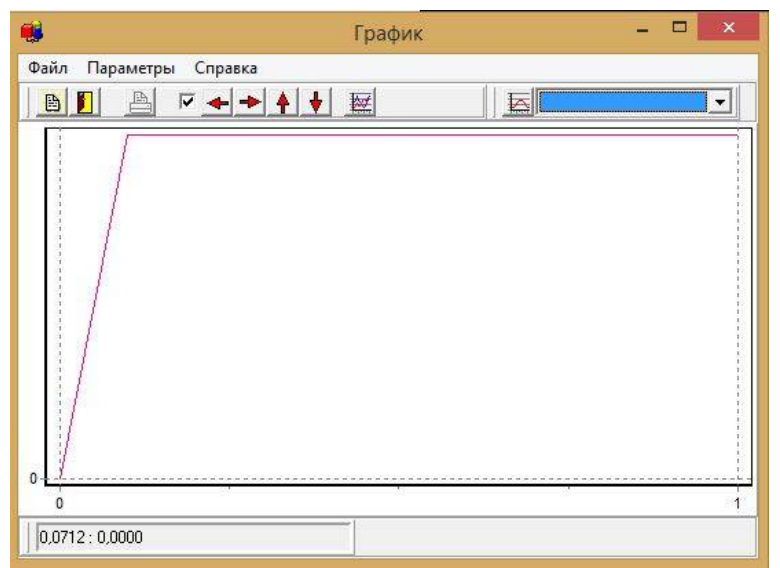

Fig. 7 The graph of forces acting on the gripper of the manipulator

Inverse problem. The manipulator is acted upon by forces $F_{1}$ and $F_{2}$ (Fig. 3), $F_{1}$ acts on the second link along the y axis, $F_{2}$ acts on the third link of the manipulator along the $\mathrm{z}$ axis. The forces are given in the form of the use (10).

$$
F_{1}\left(t, A, t_{0}, t_{1}\right)=F_{2}\left(t, A, t_{0}, t_{1}\right)=\left\{\begin{array}{l}
0, t \leq t_{0} \\
\frac{A \cdot\left(t-t_{0}\right)}{\left(t_{1}-t_{0}\right)}, t_{0}<t \leq t_{1}, \\
A, t>t_{1}
\end{array}\right.
$$

where $A$ the constant, $t_{0}, t_{1}$ the moments of change of use (10), $t$ time. For certain parameters, the force graph assumes the following form (Fig. 8):

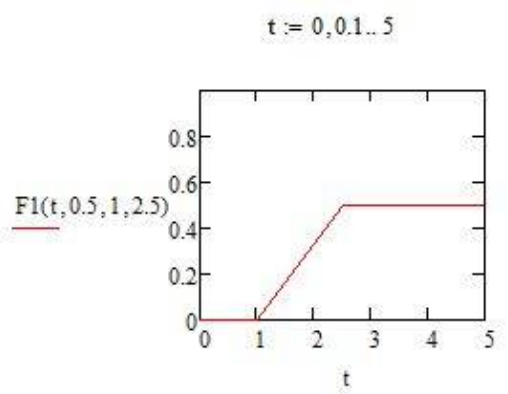

Fig. 8 Graph of function change (10)

From the given forces it is necessary to determine the trajectory, velocity and acceleration that will be required for moving the manipulator links from the initial position of Fig. 4 into the final Fig. 5. At intervals of 0 to 1 second, in 0.1 second increments. At the end of the solution of the inverse problem of dynamics, we obtain the trajectory of the grip of the manipulator at a given time interval. The results are shown in the form of a table of values in Fig. 9 and the graph of Fig. 10.

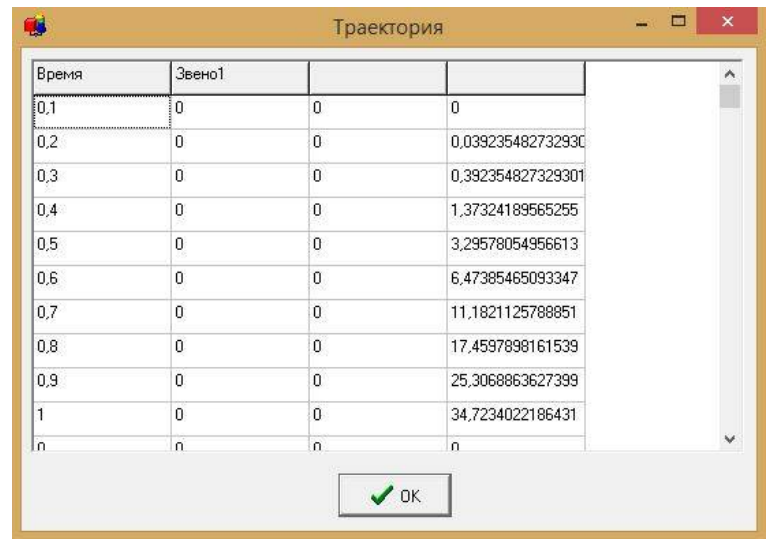

Fig. 9 Trajectory of movement of the gripper of the manipulator

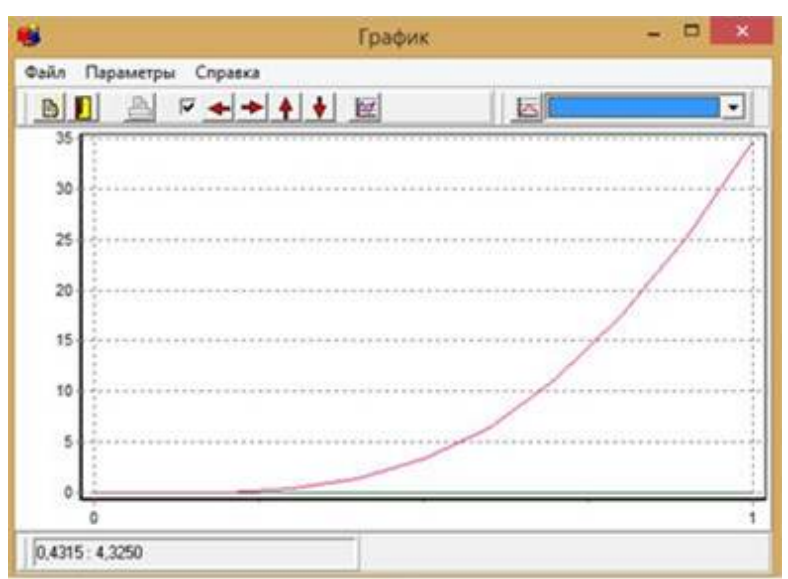

Fig. 10 Trajectory of movement of the gripper of the manipulator

\section{THE DISCUSSION OF THE RESULTS}

The developed unit allows for an arbitrary robot manipulator automatically be equations and solve the direct and inverse problem of dynamics. It finds the generalized forces that need to be specified for moving the manipulator links along a predetermined trajectory by solving the forward problem of dynamics. To calculate the path of movement of the speed and acceleration of each boom when solving the inverse problem of dynamics.

\section{CONCLUSIONS AND CONCLUSION}

As a result, the work was studied method for solving the inverse problem and the forward dynamics of the Euler-Lagrange equation based. The developed algorithm for generating the Euler-Lagrange equation for the manipulator random. There is provided a method of solving the Euler-Lagrange. The modification is made to the system modules RAUMS, to facilitate forming and solving the Euler-Lagrange equation. 
In the future it is planned to expand the capabilities of the RAUMS program. It is planned to develop and implement drive models.

\section{REFERENCES}

[1] A.N.Goritov, "Modeling of manipulative robotic systems in conditions of incomplete information about the external environment" Publishing house of the Institute of Atmospheric Optics SB RAS, 2005, pp. 276

[2] A.N.Goritov, "The architecture of the automated simulation system of robotic complexes" Software products and systems: Supplement to the journal "Problems of theory and practice of management.", 2001, pp. 17-19

[3] K.Fu, R.Gonsalis and K.Lee, "Robotics" Publishing world, 1989, pp. 624

[4] K.V.Frolova, "Mechanics of Industrial Robots: A Textbook for Universities: In 3 Books" Book 1: Kinematics and Dynamics ", Izdatelstvo obshcheia shkola, 1988 , pp. 304 